Check for updates

Cite this: RSC Adv., 2019, 9, 1949

\title{
Gelation and luminescence of lanthanide hydrogels formed with deuterium oxide $\uparrow$
}

\author{
Yusuke Zama, ${ }^{a}$ Kazushige Yanai, ${ }^{\text {b }}$ Juri Takeshita, ${ }^{\text {b }}$ Ayumi Ishii, ${ }^{\text {acde }}$ \\ Masamichi Yamanaka (D) *be and Miki Hasegawa (DD *ae
}

Gel formations and efficient lanthanide luminescence appeared in deuterium oxide $\left(\mathrm{D}_{2} \mathrm{O}\right)$ medium instead of light water $\left(\mathrm{H}_{2} \mathrm{O}\right)$, and their solvation possibilities by using luminescence lifetimes were discussed. The lanthanide ions in the hydrogel of 1 obtained by $\mathrm{H}_{2} \mathrm{O}$ (abbreviated as $\mathrm{H}_{2} \mathrm{O}-\mathrm{Ln} 1 ; \mathrm{Ln}=\mathrm{Eu}$, Tb, and $\mathrm{Gd}$ ) in our previous report act as the coupling part between neighbor molecules for the bundle structure. Here, $\mathrm{D}_{2} \mathrm{O}$ also acts as a medium to form the lanthanide-hydrogel of 1 , and increases intensities of luminescence for $\mathrm{Tb}$, because a soft crystalline state reducing resonance thermal relaxation is realized. The gel-formation and luminescence band positions of $\mathrm{Ln} 1$ in $\mathrm{D}_{2} \mathrm{O}$ corresponded to those in $\mathrm{H}_{2} \mathrm{O}$. From the observation of luminescence lifetimes in $\mathrm{H}_{2} \mathrm{O}$ and $\mathrm{D}_{2} \mathrm{O}$, the number of coordinating water molecules on $\mathrm{Eu}$ and $\mathrm{Tb}$ were estimated to be around 3 or 4 for both. The luminescence intensity of Eu1 did not increase even in $\mathrm{D}_{2} \mathrm{O}$, due to a blue shift of the excited triplet state of 1 , as compared to that in $\mathrm{H}_{2} \mathrm{O}$.

Received 14th October 2018 Accepted 19th December 2018

DOI: $10.1039 / \mathrm{c} 8 \mathrm{ra} 08504 \mathrm{~h}$

rsc.li/rsc-advances
The combination of two hetero Ln ions such as $\mathrm{Tb}$ and $\mathrm{Eu}$ with organic components can accomplish colour mixing of luminescence, and induce inter-metal energy transfer from $\mathrm{Tb}$ to Eu by UV excitation of the ligand moiety. ${ }^{7-9}$ Such a stepwise energy transfer is also useful for developing luminescent sensors or devices. Recently, we reported mixed $\mathrm{Tb} / \mathrm{Eu}$ systems in chain-structured complexes consisting of helical Ln complexes (abbreviated as $\mathrm{LnL}$; $\mathrm{Ln}=\mathrm{Eu}$ or $\mathrm{Tb}$ ) linked by analogues of 1,4-benzenedicarboxylate (abbreviated as bdc). ${ }^{\mathbf{1 0}}$ The initial compound LnL-bdc showed no inter-metal energy transfer between the energy donor (ligand) and acceptor (centre metal ion), whereas bdc substituted with $\mathrm{NH}_{2}-$ or $\mathrm{OH}$ - induced inter-metal energy transfer in the chain complexes, and resulted in reddish-orange emission even with a $1: 1$ mixture of EuL and TbL, due to its amorphous structure.

It was reported that the intensity change of lanthanide luminescence is affected by $\mathrm{O}-\mathrm{H} / \mathrm{O}-\mathrm{D}$ or $\mathrm{C}-\mathrm{H} / \mathrm{C}-\mathrm{D}$ vibrational mode, due to harmonic resonance with the luminescent level in lanthanide ions. ${ }^{\mathbf{1 1 , 1 2}}$ It is known that the quenching mechanism for trivalent $\mathrm{Eu}$ and $\mathrm{Tb}$ involves resonance of the third and fourth overtones of $\mathrm{O}-\mathrm{H}$ vibrations ( $c a .3400 \mathrm{~cm}^{-1}$ ) in $\mathrm{H}_{2} \mathrm{O}$, respectively. ${ }^{13-15}$ We recently reported the effect of deuterium on the luminescent components of $\mathrm{H} / \mathrm{D}$-phenanthroline complexes with $\mathrm{Eu}, \mathrm{Tb}$, and Tm complexes, according to the appearance of polymorphism detected by the synchrotron X-ray powder diffraction (XRPD). ${ }^{13}$

A water-soluble lanthanide complex of helicate structure with $\pi$-electronic systems and two carboxyl groups, $\mathrm{LnL}^{\mathrm{COOH}}$ also showed the change in luminescent intensity, when using $\mathrm{D}_{2} \mathrm{O}$ instead of $\mathrm{H}_{2} \mathrm{O} .{ }^{16} \mathrm{LnL}^{\mathrm{COOH}}$ retains its molecular and spectral stability in a wide $\mathrm{pH}$ range of 2.6-9.7. 
To overcome the quenching problem related to $\mathrm{Tb}$ or $\mathrm{Eu}$ in $\mathrm{H}_{2} \mathrm{O}$, some investigators reported using a capsulated molecular structure to prohibit solvent molecules from reaching Ln ions. ${ }^{17,18}$ A Ln complex ( $\mathrm{Ln}=\mathrm{Eu}, \mathrm{Tb}$, Dy, and $\left.\mathrm{Sm}\right)$ with a 2hydroxyisophthalamide derivative with a multidentate ligand has water solubility, and shows a $60 \%$ luminescence quantum yield for $\mathrm{Ln}=\mathrm{Tb}$, even in water. ${ }^{17}$ Cluster-type $\mathrm{Zn}$ complexes with Ln $\left[\mathrm{Zn}_{8}(\mathrm{ad})_{4}(\mathrm{BPDC})_{6} \mathrm{O} \cdot 2 \mathrm{Me}_{2} \mathrm{NH}_{2}, 8 \mathrm{DMF}, 11 \mathrm{H}_{2} \mathrm{O}\right](\mathrm{ad}=$ adeninate; $\mathrm{BPDC}=$ biphenyldicarboxylate; $\mathrm{DMF}=$ dimethylformamide) form the metal-organic frameworks and show Ln luminescence even in water. ${ }^{18}$

Supramolecular gels, which are formed by self-assembly of small molecules, have attracted interest in recent decades because of their wide applicability. ${ }^{\mathbf{1 9 , 2 0}}$ Some luminescent lanthanide complexes in organogels and hydrogels have been developed and reported for applications such as paintable luminescent thermo sensors and color tunable LED fibers. ${ }^{21-28}$ We also recently succeeded in obtaining bright luminescent $\mathrm{Tb}$ complexes with tris-urea-based hydrogelator 1 in $\mathrm{H}_{2} \mathrm{O} .{ }^{29}$ The gelator shown in Fig. 1 has multiple roles in hydrogel formation, by coordinating with cations such as protons, divalent calcium, trivalent Ln ions, and serving as a photo-antenna to transfer excitation energy to Ln ions. Compound $\mathbf{1}$ also shows Ln selectivity in luminescence; the hydrogel of the lanthanide complex with 1 obtained with $\mathrm{H}_{2} \mathrm{O}$ (abbreviated as $\mathrm{H}_{2} \mathrm{O}-\mathrm{Ln} \mathbf{1}$; Ln $=\mathrm{Eu}, \mathrm{Tb}$, and $\mathrm{Gd}$ ) shows different luminescence quantum yields based on the $\mathrm{Ln}$; for $\mathrm{H}_{2} \mathrm{O}-\mathrm{Eu} 1$ and $\mathrm{H}_{2} \mathrm{O}-\mathrm{Tb} 1$, the quantum yields are $<0.1 \%$ and $5.4 \%$, respectively. It was recently revealed that the abovementioned lanthanide-including hydrogels of 1 act as media for organic fluorophores such as Rhodamine 6G, ${ }^{30}$ and the interaction between the gelator and fluorophore was clearly enhanced in the luminescence as well as its lifetime.

Heavy water $\left(\mathrm{D}_{2} \mathrm{O}\right)$ has been considered to be nearly equivalent to light water $\left(\mathrm{H}_{2} \mathrm{O}\right)$, due to its physical properties in supramolecular hydrogels. Therefore, nuclear magnetic resonance (NMR) and small-angle neutron scattering (SANS) experiments have been performed in $\mathrm{D}_{2} \mathrm{O}$ as an alternative to $\mathrm{H}_{2} \mathrm{O}^{31}$ The similarities and dissimilarities of $\mathrm{D}_{2} \mathrm{O}$ and $\mathrm{H}_{2} \mathrm{O}$ are still unclear, and more study is required to clarify them. Here, we report a heavy water effect in the luminescence of a series of

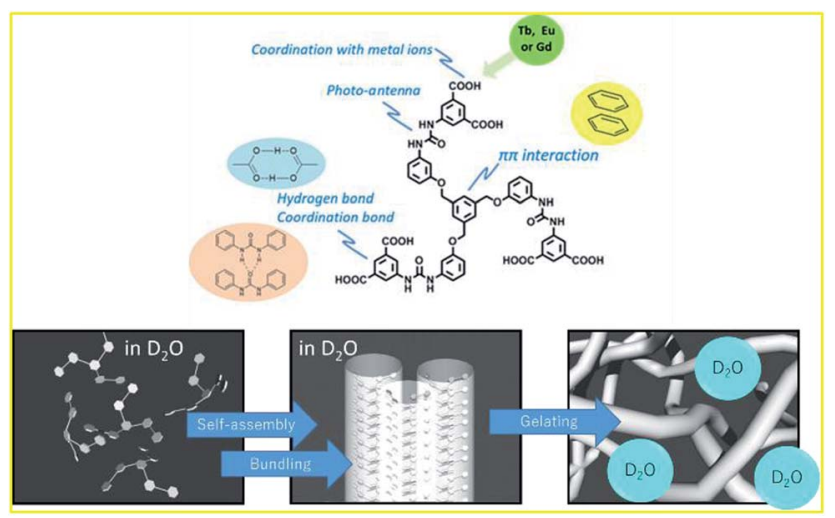

Fig. 1 Role of substances of 1 in the metal gelation (top), and schematic representation of gel formation in $\mathrm{D}_{2} \mathrm{O}$ (bottom).
$\mathrm{D}_{2} \mathrm{O}-\mathrm{Ln} 1$ based on measurements of electronic absorption, excitation, and luminescence spectra. Furthermore, the intermetal energy transfer between $\mathrm{Eu}$ and $\mathrm{Tb}$ was induced in $\mathrm{D}_{2} \mathrm{O}$ Ln1. The luminescence lifetime and quantum yield were also measured to evaluate the luminescence efficiency in these hydrogels with Ln.

\section{Results and discussion}

\section{Hydrogel formation and structure of $\mathrm{D}_{2} \mathrm{O}-\mathrm{Ln} 1$}

Viscoelastic features of hydrogels $D_{2} \mathrm{O}-\mathrm{Ln} 1$ and $\mathrm{H}_{2} \mathrm{O}-\mathrm{Ln} 1$. The viscoelastic properties of the hydrogels $\mathrm{D}_{2} \mathrm{O}-\mathrm{Tb} 1$ and $\mathrm{H}_{2} \mathrm{O}$ $\mathrm{Tb} \mathbf{1}$ were measured using a rheometer. The concentrations of 1 and $\mathrm{Tb}(\mathrm{OTf})_{3}$ in hydrogels were fixed at $10 \mathrm{mM}$ and $30 \mathrm{mM}$, respectively. The hydrogels $\mathrm{D}_{2} \mathrm{O}-\mathrm{Tb} 1$ and $\mathrm{H}_{2} \mathrm{O}$-Tb1 showed similar linear viscoelastic frequency sweep responses (Fig. 2a). Both the storage modulus $\left(G^{\prime}\right)$ and the loss modulus $\left(G^{\prime \prime}\right)$ were almost independent of the frequency from 0.1 to $10 \mathrm{rad} \mathrm{s}^{-1}$. For both samples, $G^{\prime}$ was about twelve times larger than $G^{\prime \prime}$. The $G^{\prime}$ values for the hydrogels $\mathrm{D}_{2} \mathrm{O}-\mathrm{Tb} 1$ and $\mathrm{H}_{2} \mathrm{O}-\mathrm{Tb} 1$ at $1.0 \mathrm{rad} \mathrm{s}^{-1}$ were 42.8 and $42.7 \mathrm{kPa}$, respectively. Previously, we reported that the $G^{\prime}$ value for $\mathrm{H}_{2} \mathrm{O}-\mathrm{Tb} 1$ at $1.0 \mathrm{rad} \mathrm{s}^{-1}$ was $8.1 \mathrm{kPa} .^{29}$ This result was obtained by measuring a relatively large hydrogel using a flat plate geometry $(25 \mathrm{~mm})$. Several pieces of the hydrogel were present in the measured sample. In the present study, we prepared the hydrogel in an aluminium cup $(14 \mathrm{~mm}$ diameter) as a single continuous sample. ${ }^{30}$ This unbroken sample exhibited a larger $G^{\prime}$ value. As shown in Fig. 2b, strain amplitude sweeps of $\mathrm{D}_{2} \mathrm{O}-\mathrm{Tb} 1$ and $\mathrm{H}_{2} \mathrm{O}-\mathrm{Tb} 1$ demonstrated elastic responses. The linear viscoelastic region of $\mathrm{D}_{2} \mathrm{O}-\mathrm{Tb} 1$ was extensive, in comparison to that of $\mathrm{H}_{2} \mathrm{O}-\mathrm{Tb} 1$.

SEM and EDX measurement of hydrogels of Tb/Eu1. Energy dispersive X-ray (EDX) spectroscopy of the xerogel produced from a 1:1 mixture of hydrogels containing Eu1 and Tb1 is shown in Fig. 3. The peaks for Eu and Tb appear at 1.15 and 1.25 $\mathrm{keV}$, respectively. The component ratios of elements are $\mathrm{C}$, $73.8 \%$; N, 9.8\%; O, 13.8\%; Tb, 1.9\%; and $\mathrm{Eu}, 1.7 \%$. Tb and $\mathrm{Eu}$ are localized on the fiber in equal amounts, which is consistent with the results of a previous study. ${ }^{29}$

\section{Luminescence properties of $\mathrm{D}_{2} \mathrm{O}-\mathrm{Ln} 1$}

Luminescence spectra of $\mathrm{D}_{2} \mathrm{O}$-Tb1 and $\mathrm{D}_{2} \mathrm{O}$-Eu1. $\mathrm{D}_{2} \mathrm{O}$-Tb1, a gel complex with trivalent $\mathrm{Tb}$ ions, was obtained as a hydrogel. Under UV light, the luminescence was a greenish colour to the naked eye. Electronic absorption spectra of $\mathrm{D}_{2} \mathrm{O}-\mathrm{Ln} 1(\mathrm{Ln}=\mathrm{Tb}$,
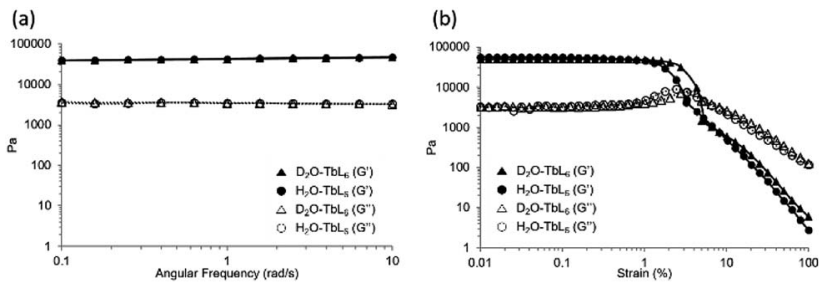

Fig. $2 G^{\prime}$ and $G^{\prime \prime}$ values for $\mathrm{D}_{2} \mathrm{O}$-Tb1 (triangles) and $\mathrm{H}_{2} \mathrm{O}-\mathrm{Tb} 1$ (circles) (a) with a frequency sweep; (b) with a strain sweep. 

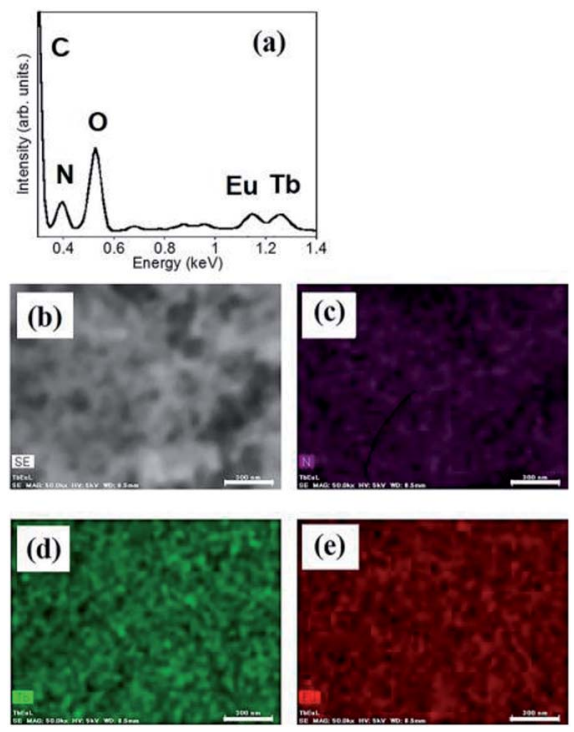

Fig. 3 EDX spectrum (a), SEM image (b), and element maps ((c) N; (d) $\mathrm{Tb}$ and (e) Eu) of the xerogel obtained from hydrogels by mixing of Eu1 and Tb1. Scale bar shows $100 \mu \mathrm{m}$.

$\mathrm{Eu}$, and Gd) were measured (Fig. 4) to compare the electronic properties with those found previously for $\mathrm{H}_{2} \mathrm{O}-\mathrm{Ln} 1$. The bands at 288 and $323 \mathrm{~nm}$ and their shapes for $\mathrm{D}_{2} \mathrm{O}-\mathrm{Ln} 1$ correspond well to those for $\mathrm{H}_{2} \mathrm{O}$-Ln1, meaning that their $\pi$-electronic states with $\mathrm{D}_{2} \mathrm{O}$ completely matched those with $\mathrm{H}_{2} \mathrm{O}$. Additionally, the $\mathrm{D}_{2} \mathrm{O}$ hydrogel of $\mathbf{1}$ without metal also shows the corresponding absorption band. This means that these metallo-hydrogels have similar $\pi$-electronic structures.

Luminescence spectra of $\mathrm{D}_{2} \mathrm{O}-\mathrm{Tb} \mathbf{1}$ and $\mathrm{D}_{2} \mathrm{O}$-Eu1 are shown in Fig. 5. $\mathrm{D}_{2} \mathrm{O}-\mathrm{Tb} 1$ shows sharp luminescence bands at 489,544 , 583, and $621 \mathrm{~nm}$ assigned to the ${ }^{5} \mathrm{D}_{4} \rightarrow{ }^{7} \mathrm{~F}_{6},{ }^{5} \mathrm{D}_{4} \rightarrow{ }^{7} \mathrm{~F}_{5},{ }^{5} \mathrm{D}_{4} \rightarrow$ ${ }^{7} \mathrm{~F}_{4}$, and ${ }^{5} \mathrm{D}_{4} \rightarrow{ }^{7} \mathrm{~F}_{3}$ transitions, respectively. The excitation spectra monitored at these luminescence band positions correspond to those for the electronic absorption spectra (Fig. $\mathrm{S} 1 \dagger$ ). Luminescence and excitation bands for $\mathrm{D}_{2} \mathrm{O}-\mathrm{Tb} 1$ reproduced those of $\mathrm{H}_{2} \mathrm{O}-\mathrm{Tb} 1$, indicating that gelator 1 coordinates to the Ln ions while maintaining a suitable distance, to induce excitation energy transfer even in $\mathrm{D}_{2} \mathrm{O}-\mathrm{Tb} \mathbf{1}$.

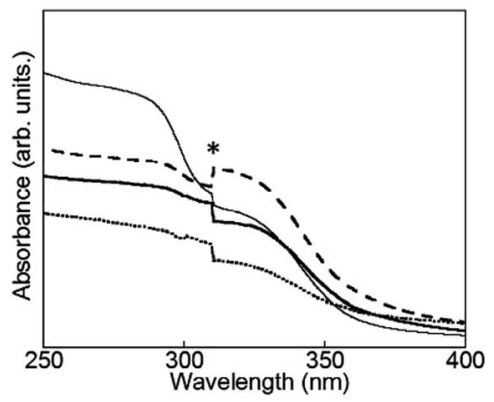

Fig. 4 Electronic absorption spectra of $\mathrm{D}_{2} \mathrm{O}-\mathrm{Tb} 1$ (solid line), $\mathrm{D}_{2} \mathrm{O}-\mathrm{Eu} 1$ (dotted line), $\mathrm{D}_{2} \mathrm{O}-\mathrm{Gd} 1$ (dashed line), and $\mathrm{D}_{2} \mathrm{O}-\mathrm{H} 1$ (thin line). * Due to apparatus.
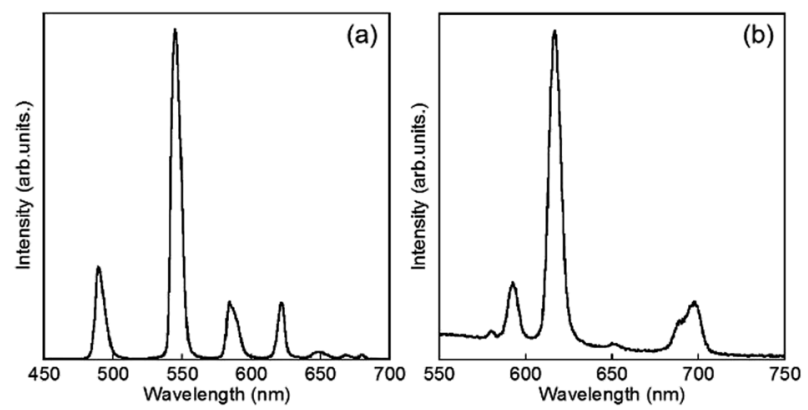

Fig. 5 Luminescence spectra of $\mathrm{D}_{2} \mathrm{O}-\mathrm{Tb} 1(\mathrm{a})$ and $\mathrm{D}_{2} \mathrm{O}-\mathrm{Eu} 1$ (b). $\lambda_{\text {ex }}=$ $323 \mathrm{~nm}$.

To evaluate the luminescence of $\mathrm{D}_{2} \mathrm{O}-\mathrm{Tb} 1$ quantitatively, luminescence quantum yields and lifetimes were measured and are summarized in Table 1 . From the luminescence decay curves monitored at $545 \mathrm{~nm}$ for both $\mathrm{D}_{2} \mathrm{O}-\mathrm{Tb} 1$ and $\mathrm{H}_{2} \mathrm{O}-\mathrm{Tb} 1$ (Fig. 6), it is clear that the luminescence decay for $\mathrm{Tb}$ in $\mathrm{D}_{2} \mathrm{O}-\mathrm{Tb} 1$ was much slower than that for $\mathrm{H}_{2} \mathrm{O}-\mathrm{Tb} 1$. This indicates that the luminescence rate is influenced by the vibrational mode of the water medium. Based on the decomposition of the decay curves, $\mathrm{D}_{2} \mathrm{O}-\mathrm{Tb1}$ has at least three luminescent components, whereas the corresponding components in $\mathrm{H}_{2} \mathrm{O}-\mathrm{Tb} 1$ were shorter than those in $\mathrm{D}_{2} \mathrm{O}$-Tb1. Surprisingly, the longest luminescence lifetime was for $\mathrm{D}_{2} \mathrm{O}-\mathrm{Tb} 1$. A typical value in terbium complexes in the solid state is $c a$. 1.5-1.7 ms. Thus, deuterium oxide accelerates $\mathrm{Tb}$ luminescence efficiently in the gel of $\mathrm{D}_{2} \mathrm{O}-\mathrm{Tb} \mathbf{1}$, and this is known as the heavy water effect. The luminescence quantum yield for $\mathrm{D}_{2} \mathrm{O}-\mathrm{Tb} 1$ is twice that for $\mathrm{H}_{2} \mathrm{O}-\mathrm{Tb} 1$, which also supports the above conclusion. Thus, heavy water acts as an accelerator for $\mathrm{Tb}$ luminescence even in hydrogels.

$\mathrm{D}_{2} \mathrm{O}-\mathrm{Eu} 1$ also exhibits luminescence bands at 580, 592, 616, 650 , and $697 \mathrm{~nm}$, which are assigned to the ${ }^{5} \mathrm{D}_{0} \rightarrow{ }^{7} \mathrm{~F}_{0},{ }^{5} \mathrm{D}_{0} \rightarrow$ ${ }^{7} \mathrm{~F}_{1},{ }^{5} \mathrm{D}_{0} \rightarrow{ }^{7} \mathrm{~F}_{2},{ }^{5} \mathrm{D}_{0} \rightarrow{ }^{7} \mathrm{~F}_{3}$, and ${ }^{5} \mathrm{D}_{0} \rightarrow{ }^{7} \mathrm{~F}_{4}$ transitions, respectively. These band positions are mostly the same for $\mathrm{H}_{2} \mathrm{O}$-Eu1 (Fig. $\mathrm{S} 2 \dagger$ ). The luminescence quantum yields for $\mathrm{Eu}$ in $\mathrm{D}_{2} \mathrm{O}$-Eu1 and $\mathrm{H}_{2} \mathrm{O}$-Eu1 are quite low (Table 1). The luminescence decay profile for $\mathrm{D}_{2} \mathrm{O}$-Eu1 was divided into two components, whose lifetimes are longer than those of $\mathrm{H}_{2} \mathrm{O}$-Eu1. This is in contrast to the two gel complexes with $\mathrm{Tb}$, and may be also due to the vibrational mode of water as the medium.

The number of coordinating water molecules on Ln ions in hydrogels can be estimated using the experimental Horrocks' equation for the lifetime: ${ }^{32-35}$

Table 1 Luminescence quantum yields and lifetimes for $\mathrm{D}_{2} \mathrm{O}-\mathrm{Tb} 1$ and $\mathrm{D}_{2} \mathrm{O}$-Eu1 compared to those with light water ${ }^{28}$

\begin{tabular}{lllll}
\hline & & \multicolumn{3}{l}{ Lifetimes $/ \mathrm{ms}(\mathrm{amp}) ; \lambda_{\mathrm{ex}}=340 \mathrm{~nm}^{c}$} \\
\cline { 3 - 5 } & Quantum yields & $\tau_{1}$ & $\tau_{2}$ & $\tau_{3}$ \\
\hline $\mathrm{D}_{2} \mathrm{O}-\mathrm{Tb1}$ & $10 \%^{a}$ & $0.20(14 \%)$ & $0.83(41 \%)$ & $1.7(45 \%)$ \\
$\mathrm{H}_{2} \mathrm{O}-\mathrm{Tb1}$ & $5.4 \%^{a}$ & $0.08(8 \%)$ & $0.37(37 \%)$ & $0.70(54 \%)$ \\
$\mathrm{D}_{2} \mathrm{O}-\mathrm{Eu} 1$ & $<0.1 \%^{b}$ & $0.32(25 \%)$ & $1.06(75 \%)$ & - \\
$\mathrm{H}_{2} \mathrm{O}-\mathrm{E} 1$ & $<0.1 \%^{b}$ & $0.10(23 \%)$ & $0.22(77 \%)$ & - \\
${ }^{a} \lambda_{\text {obs }}=450-700 \mathrm{~nm}^{b}{ }^{b} \lambda_{\text {obs }}=550-750 \mathrm{~nm} .{ }^{c} \lambda_{\text {mon }}=545$ and $616 \mathrm{~nm}$ for \\
Tb and Eu, respectively.
\end{tabular}




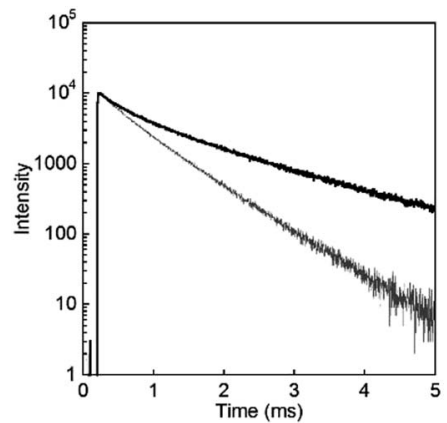

Fig. 6 Luminescence decay profiles for $\mathrm{D}_{2} \mathrm{O}-\mathrm{Tb} 1$ (solid line) and $\mathrm{H}_{2} \mathrm{O}$ $\mathrm{Tb} 1$ (thin grey line). $\lambda_{\text {mon }}=545 \mathrm{~nm}$ and $\lambda_{\mathrm{ex}}=323 \mathrm{~nm}$.

$$
q^{\mathrm{Ln}}=x\left(1 / \tau_{\mathrm{H}_{2} \mathrm{O}}-1 / \tau_{\mathrm{D}_{2} \mathrm{O}}\right)
$$

where, $q^{\mathrm{Ln}}$ is the number of water molecules coordinating to a Ln ion, $x$ is a fixed number (1.02 for Eu and 4.2 for Tb), and $\tau_{\mathrm{H}_{2} \mathrm{O}}$ and $\tau_{\mathrm{D}_{2} \mathrm{O}}$ are the average luminescence lifetimes (eqn (2)) observed in $\mathrm{H}_{2} \mathrm{O}$ and $\mathrm{D}_{2} \mathrm{O}$, respectively: ${ }^{36}$

$$
\tau=\left(a_{1} \tau_{1}^{2}+a_{2} \tau_{2}^{2}\right) /\left(a_{1} \tau_{1}+a_{2} \tau_{2}\right)
$$

Here, $\tau_{n}$ and $a_{n}$ are the $n^{\text {th }}$ observed luminescence lifetime and its amplitude, respectively. We used the values in Table 1, and $q_{\mathrm{Tb}}$ and $q_{\mathrm{Eu}}$ were calculated as 3.7 and 3.1, respectively. Both metal ions in hydrogels will bind three or four water molecules. Thus, the O-H or O-D vibrational mode will directly affect the energy transfer efficiency or excitation energy relaxation, including non-radiative processes in these hydrogels.

To clarify the energy relaxation process for the water vibrational mode, ligand-centred emissions such as fluorescence and phosphorescence were observed. Fig. 7 shows fluorescence spectra of $\mathrm{D}_{2} \mathrm{O}-\mathrm{H} \mathbf{1}$ and phosphorescence spectra of $\mathrm{D}_{2} \mathrm{O}-\mathrm{Gd} \mathbf{1}$. The compounds $\mathrm{D}_{2} \mathrm{O}-\mathrm{H} 1$ and $\mathrm{D}_{2} \mathrm{O}-\mathrm{Tb} 1$ also formed stable hydrogels similar to $\mathrm{D}_{2} \mathrm{O}$-Tb1. The use of $\mathrm{Gd}$ ion instead of a luminescent Ln ion is the conventional means for the determination of the singlet and triplet states as the energy donor in the $\pi$-electron system. ${ }^{37}$ In our previous report, the fluorescence and phosphorescence bands for 1 using the hydrogel $\mathrm{H}_{2} \mathrm{O}-\mathrm{Gd} \mathbf{1}$ were at 363 and $465 \mathrm{~nm}$, respectively. ${ }^{29}$ The corresponding bands appear at $363\left(27500 \mathrm{~cm}^{-1}\right)$ and $477\left(21000 \mathrm{~cm}^{-1}\right) \mathrm{nm}$,

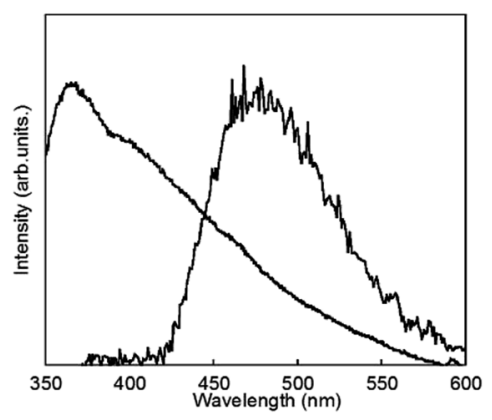

Fig. 7 Fluorescence spectrum of $\mathrm{D}_{2} \mathrm{O}-\mathrm{H} 1$ (shorter wavelength band and phosphorescence spectrum at $77 \mathrm{~K}$ ) and of $\mathrm{D}_{2} \mathrm{O}$-Gd1 (longer wavelength band) localized on $1\left(\lambda_{\mathrm{ex}}=323 \mathrm{~nm}\right)$. respectively, in the present compounds. The 477 band acts as an energy donor to $\mathrm{Tb}$ or $\mathrm{Eu}$ in hydrogels. The phosphorescence band position for $\mathrm{D}_{2} \mathrm{O}-\mathrm{Gd} \mathbf{1}$ is lower than that for $\mathrm{H}_{2} \mathrm{O}-\mathrm{Gd} \mathbf{1}$ by $12 \mathrm{~nm}$, and may affect the energy transfer efficiency of $\mathrm{D}_{2} \mathrm{O}-\mathrm{Eu} 1$ which has acceptor bands around 394 or $416 \mathrm{~nm} .{ }^{38}$ Additionally, $\mathrm{H}_{2} \mathrm{O}$-Tb1 and $\mathrm{D}_{2} \mathrm{O}$ - $\mathrm{Tb} 1$ show broad luminescence bands in laser Raman spectra at $637\left(15700 \mathrm{~cm}^{-1}\right)$ and $625\left(16000 \mathrm{~cm}^{-1}\right) \mathrm{nm}$, respectively (Fig. 8). These bands do not correspond to the fluorescence and phosphorescence in Fig. 6, suggesting that the luminescence bands are caused by strong intermolecular interactions by the formation of supramolecular hydrogels. It is noteworthy that the band position for self-assemblies of the gelator covers the Eu luminescence bands. In other words, the $\mathrm{O}-\mathrm{D}$ vibrational mode intensified the luminescence of $\mathrm{Tb}$ in $\mathrm{D}_{2} \mathrm{O}$ - $\mathrm{Tb} \mathbf{1}$. In contrast, the weakened luminescence for $\mathrm{Eu}$ in $\mathrm{D}_{2} \mathrm{O}$-Eu1 was caused by the $\mathrm{O}-\mathrm{D}$ vibration in deuterium oxide and by superimposition of the emission of self-assembly in the gelator and luminescence of Eu.

Luminescence spectra of $\mathbf{D}_{\mathbf{2}} \mathbf{O}$-Tb/Eu1. Metal-to-metal excitation energy transfer between $\mathrm{Tb}$ and Eu suggests metal-metal interaction, by the measurement of their luminescence. We prepared the 1:1 mixed hydrogels of Eu and Tb with 1 in $\mathrm{H}_{2} \mathrm{O}$ and $\mathrm{D}_{2} \mathrm{O}$, abbreviated as $\mathrm{H}_{2} \mathrm{O}-\mathrm{Tb} / \mathrm{Eu} 1$ and $\mathrm{D}_{2} \mathrm{O}-\mathrm{Tb} / \mathrm{Eu} 1$, respectively. From the EDX measurement as described above, nitrogen in 1 was also found at the similar position with the metal ions. This supports luminescence effects such as inter-metal interactions between $\mathrm{Tb}$ and $\mathrm{Eu}$ in the mixed hydrogel of Eu1 and Tb1.

Fig. 9 shows luminescence spectra of hydrogels containing $\mathrm{Tb}$ and Eu ions in a $1: 1$ ratio, which are abbreviated as $\mathrm{H}_{2} \mathrm{O}-\mathrm{Tb} /$ Eu1 and $\mathrm{D}_{2} \mathrm{O}-\mathrm{Tb} / \mathrm{Eu} 1$ for light and heavy water, respectively. Bands at 544, 545, 584, 585, 620, 650(sh), and 679(sh) nm, assigned to the overlap of ff transitions of $\mathrm{Tb}^{3+}$ and $\mathrm{Eu}^{3+}$, are observed for $\mathrm{H}_{2} \mathrm{O}-\mathrm{Tb} / \mathrm{Eu} 1$. The corresponding bands for $\mathrm{D}_{2} \mathrm{O}-\mathrm{Tb} /$ Eu1 appear at 544, 584, 590, 615, 649, and $696 \mathrm{~nm}$, respectively.

The luminescence decay profile of $\mathrm{H}_{2} \mathrm{O}-\mathrm{Tb} / \mathrm{Eu} 1$ was monitored at both the emission band positions of $\mathrm{Tb}$ and $\mathrm{Eu}$ (Fig. S3 $\uparrow$ and Table 2). The decay curve of $\mathrm{H}_{2} \mathrm{O}-\mathrm{Tb} / \mathrm{Eu} 1$ monitored at $545 \mathrm{~nm}$ for $\mathrm{Tb}$ was divided into three luminescent components, and that at $616 \mathrm{~nm}$ for Eu into two. In the luminescence decay profile monitored at $616 \mathrm{~nm}$ for $\mathrm{H}_{2} \mathrm{O}-\mathrm{Tb} / \mathrm{Eu} 1$, the luminescence rise curve for $\mathrm{Eu}$ was obtained and resulted in the time as 0.15 $\mathrm{ms}$ because the time range corresponds to the decay time of

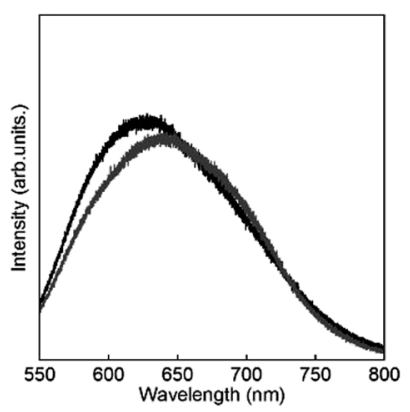

Fig. 8 Luminescence spectra of $\mathrm{D}_{2} \mathrm{O}-\mathrm{Tb} 1$ (black line) and $\mathrm{H}_{2} \mathrm{O}-\mathrm{Tb} 1$ (grey line) observed by Raman spectroscopy ( $\lambda_{\mathrm{ex}}=532 \mathrm{~nm}$ ). 


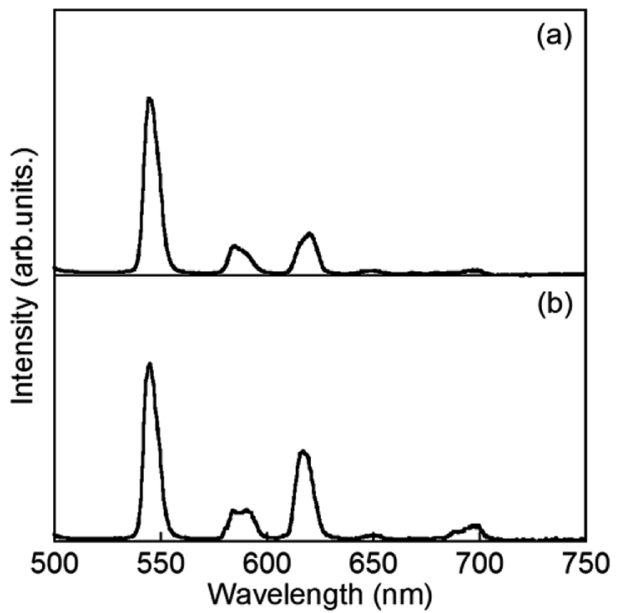

Fig. 9 Luminescence spectra of $\mathrm{H}_{2} \mathrm{O}-\mathrm{Tb} /$ Eu1 and $\mathrm{D}_{2} \mathrm{O}-\mathrm{Tb} / \mathrm{Eu} 1\left(\lambda_{\mathrm{ex}}=\right.$ $323 \mathrm{~nm})$

$0.19 \mathrm{~ms}$ for $\mathrm{Tb}$. These results are consistent with $\mathrm{Tb}^{3+}$ ions transferring the excitation energy to $\mathrm{Eu}^{3+}$ in the mixed metal hydrogels. It also demonstrated that $\mathrm{Tb}$ and $\mathrm{Eu}$ would exist sufficiently close together, so as to promote metal-to-metal energy transfer in gels. ${ }^{39}$ Similar metal-to-metal energy transfer was also observed in the xerogel obtained from hydrogels with both $\mathrm{Tb}$ and $\mathrm{Eu}$. It should note to worth that the number of luminescent decay component of $\mathrm{Eu}$ in $\mathrm{D}_{2} \mathrm{O}-\mathrm{Tb} / \mathrm{Eu} 1$ is one; however, the rise in luminescence lifetime of $0.29 \mathrm{~ms}$ is higher than that in $\mathrm{H}_{2} \mathrm{O}-\mathrm{Tb} / \mathrm{Eu1}$. It suggests that the metal-to-metal energy transfer from $\mathrm{Tb}$ to $\mathrm{Eu}$ in the gel with $\mathrm{D}_{2} \mathrm{O}$ is more efficient than that with $\mathrm{H}_{2} \mathrm{O}$.

The total luminescence quantum yield of Tb/Eu1 was observed in the $\mathrm{Tb}$ and Eu emission region (500-750 nm). The quantum yield of $\mathrm{D}_{2} \mathrm{O}-\mathrm{Tb} / \mathrm{Eu} 1$ was $2 \%$, which is higher than that of $\mathrm{H}_{2} \mathrm{O}-\mathrm{Tb} / \mathrm{Eu} 1$ and xerogel $(<0.1 \%)$. These trends support the above discussion.

The xerogel obtained from hydrogels of Tb/Eu1 showed the luminescence spectra and quantitative properties similar to those of the initial compound. It is also consistent that heavy water in this hydrogel system plays a role in enhancing lanthanide luminescence and inter-metal energy transfer. The increase in the $\varphi$ value for the xerogel at $77 \mathrm{~K}$ means the increase in forwarded energy transfer from 1 to the $\mathrm{Tb}$ ion, because of the existence of thermal equilibrium between the donor and accepter levels in the Tb complexes. ${ }^{7,9,40}$
The energy diagram for Tb/Eu1 systems is shown in Fig. 10. The excited triplet state of $\mathbf{1}$ acts as an energy donor to the terbium with less contribution of backward energy transfer. ${ }^{29}$ However, the energy difference between $\mathbf{1}$ and the Eu ion is not suitable for efficient energy transfer. The mixing of $\mathrm{Tb}$ and $\mathrm{Eu}$ in the hydrogel formed with $\mathrm{D}_{2} \mathrm{O}$ accomplished acceleration of $\mathrm{Eu}$ emission by $\mathrm{Tb}$, through metal-metal energy transfer in $\mathrm{D}_{2} \mathrm{O}$ $\mathrm{Tb} / \mathrm{Eu} 1$.

\section{Experimental}

\section{Sample preparation}

The gelator 1 and the gel containing $\mathrm{Ln}$ ions and $\mathrm{D}_{2} \mathrm{O}$ were prepared as given in the previous paper using $\mathrm{H}_{2} \mathrm{O} \cdot{ }^{29,30}$ Lanthanide-containing hydrogels were obtained from light water and $\mathrm{D}_{2} \mathrm{O}$ (Isotech Inc.), and lanthanide nitrates were used as the sources of metal ions ( $\mathrm{Ln}=\mathrm{Eu}, \mathrm{Tb}$ and $\mathrm{Gd})$. The $1: 1$ mixed hydrogels using $\mathrm{Eu}$ and $\mathrm{Tb}$ were also obtained by the mixing of europium nitrate and terbium nitrate equivalently. To obtain a metal-free hydrogel, hydrochloride and $\mathrm{D}_{2} \mathrm{O}$ were used.

\section{Apparatus}

Rheological measurements were carried out on a DHR-2 rheometer (TA Instruments), using a flat plate geometry (8 $\mathrm{mm})$. Electronic absorption and luminescence spectra were

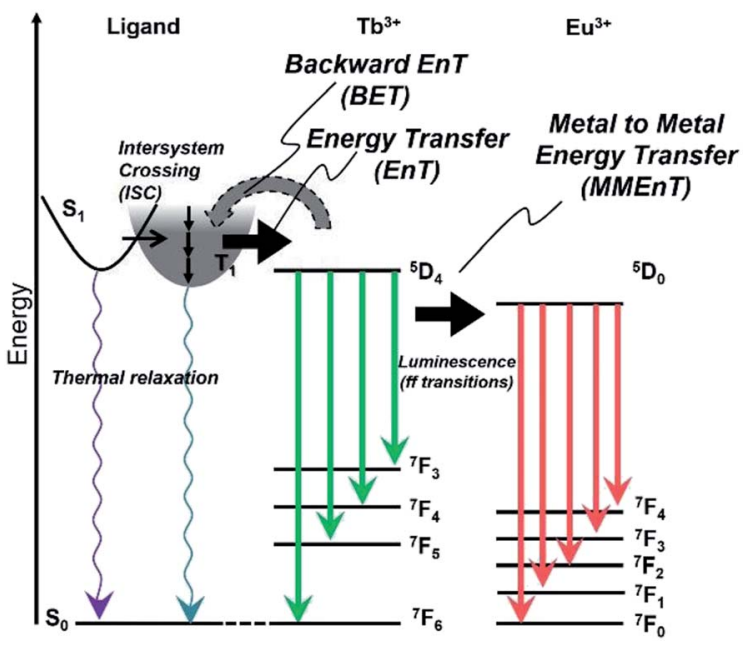

Fig. 10 Schematic representation of energy diagram for 1 and Tbsensitizing of the Eu luminescence in hydrogels.

Table 2 Photophysical properties of mixed-metal systems in hydro/xerogels. $\lambda_{\mathrm{ex}}=340 \mathrm{~nm}^{d}$

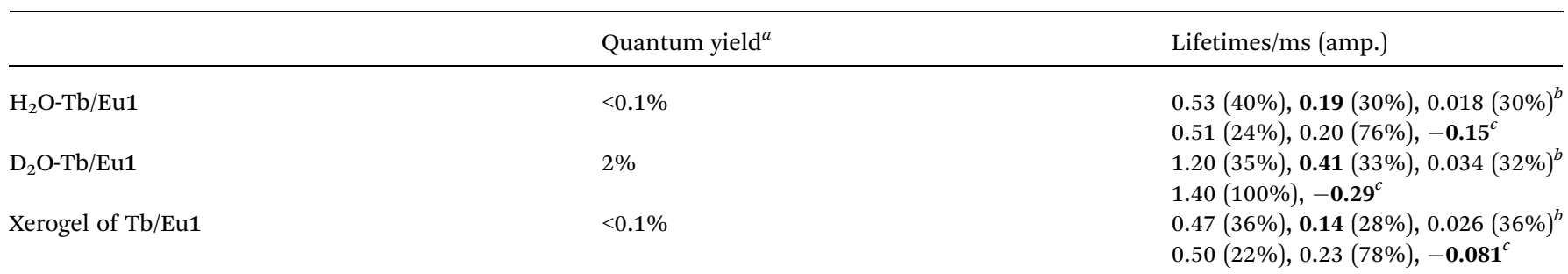

${ }^{a} \lambda_{\text {obs }}=450-700 \mathrm{~nm} .{ }^{b} \lambda_{\text {mon }}=545 \mathrm{~nm}$ for Tb. ${ }^{c} \lambda_{\text {mon }}=616 \mathrm{~nm}$ for Eu. ${ }^{d}$ Minus values in lifetimes represent the rise times of luminescence. 
recorded using a Shimadzu 3600S UV-VIS-NIR spectrophotometer and a Horiba Jovin-Yvon Fluorolog 3-22 spectrofluorometer, respectively. Luminescence quantum yields and lifetimes were measured by a Hamamatsu 9920-02 absolute photoluminescence quantum yield spectrometer and Quantaurus tau fluorescence lifetime spectrometer, respectively. SEM images were obtained using an Ultra-55 scanning electron microscope (Carl Zeiss AG) equipped with a secondary in-lens electron detector, together with a QUANTAX detector (Bruker Corporation) for EDX. The micro Raman spectra were obtained using Renishow via a Reflex spectrometer equipped with a $532 \mathrm{~nm}$ diode laser, calibrated to the $520.5 \mathrm{~cm}^{-1}$ line of silicon.

\section{Conclusion}

Three types of lanthanide ions aggregate to form hydrogels with $\mathbf{1}$, even in heavy water. The viscoelastic property of the hydrogel $\mathrm{D}_{2} \mathrm{O}-\mathrm{Tb} 1$ was identical to that of $\mathrm{H}_{2} \mathrm{O}-\mathrm{Tb1}$, whereas deuterium oxide in the gel $\mathrm{D}_{2} \mathrm{O}$-Tb1 enhanced the efficiency of ff emission of $\mathrm{Tb}$ ion. There was no influence on the luminescence of $\mathrm{D}_{2} \mathrm{O}$ Eu1 by heavy water. The fluorescence and phosphorescence bands localized on 1 were observed using $\mathrm{D}_{2} \mathrm{O}-\mathrm{H} \mathbf{1}$ (metal free; prepared by using hydrochloride) and $\mathrm{D}_{2} \mathrm{O}-\mathrm{Gd} \mathbf{1}$, respectively. Raman spectra supported the weak luminescence of $\mathrm{Eu}$ in this system. Finally, Eu emission was enhanced in the mixed hydrogel $\mathrm{D}_{2} \mathrm{O}-\mathrm{Tb} / \mathrm{Eu} 1$ through inter-metal energy transfer. These results will provide new aspects for designing hydrogelators in heavy water.

\section{Conflicts of interest}

There are no conflicts to declare.

\section{Acknowledgements}

Authors acknowledge Grants-in-Aid for Scientific Research on Innovative Areas: KAKENHI "Soft Crystals (Area Number: 2903)", No. 17H06374 for M. H., "Fusion Materials (Area Number: 2206)”, No. 25107730 for M. H., and No. 25107713 for M. Y. from Japan Society for the Promotion of Science, and Challenging Exploratory Research Centre Project for Private University and a matching fund subsidy from MEXT (2013-2017 for M. H.). M. H. also thanks Izumi Science and Technology Foundation, and Aoyama Gakuin University Soken Project for their support. EDX and SEM images measurements were supported at Centre for Instrumental Analysis, College of Science and Engineering, Aoyama Gakuin University.

\section{References}

1 J. L. Kropp and M. W. Windsor, J. Phys. Chem., 1963, 39, 2769.

2 J. I. Bruce, M. P. Lowe and D. Parker, in The Chemistry of Contrast Agents in Medical Magnetic Resonance Imaging, ed.

A. E. Merbach and E. Tóth, John Wiley and Sons, 2001, ch 11, p. 437.

3 J. C. Bünzli and C. Piguet, Chem. Soc. Rev., 2005, 34, 1048. 4 S. V. Eliseeva and J. C. Bünzli, Chem. Soc. Rev., 2010, 39, 189.
5 J. C. Bünzli, Chem. Rev., 2010, 110, 2729.

6 L. Prodi, M. Maestri, R. Ziessel and V. Balzani, Inorg. Chem., 1991, 30, 3798.

7 M. Latva, H. Takalo, V. Mukkala, C. Matachescu, J. C. Rodriguez-Ubis and J. Kankare, J. Lumin., 1997, 75, 149.

8 K. Miyata, Y. Konno, T. Nakanishi, M. Kobayashi, M. Kato, K. Fushimi and Y. Hasegawa, Angew. Chem., Int. Ed., 2013, $52,6413$.

9 M. Hatanaka, Y. Hirai, Y. Kitagawa, T. Nakanishi, Y. Hasegawa and K. Morokuma, Chem. Sci., 2017, 8, 423.

10 S. Sato, A. Ishii, C. Yamada, J. Kim, C. H. Song, A. Fujiwara, M. Takata and M. Hasegawa, Polym. J., 2015, 47, 195.

11 A. Beeby, I. M. Clarkson, R. S. Dickins, S. Faulkner, D. Parker, L. Royle, A. S. de Sousa, J. A. G. Williams and M. Woods, J. Chem. Soc., Perkin Trans. 2, 1999, 3, 493.

12 R. S. Dickins, D. Parker, A. S. de Sousa and J. A. G. Williams, Chem. Commun., 1996, 6, 697.

13 S. Ogata, A. Ishii, C. L. Lu, T. Kondo, N. Yajima and M. Hasegawa, J. Photochem. Photobiol., A, 2017, 334, 55.

14 W. D. Horrocks and D. R. Sudnick, Acc. Chem. Res., 1981, 14, 384.

15 G. Stein and E. Würzberg, J. Chem. Phys., 1975, 62, 208.

16 S. Ogata, T. Shimizu, T. Ishibashi, Y. Ishiyone, M. Hanami, M. Ito, A. I. S. Kawaguchi, K. Sugimoto and M. Hasegawa, New J. Chem., 2017, 41, 6385.

17 S. Petoud, S. M. Cohen, J. C. Bünzli and K. N. Raymond, J. Am. Chem. Soc., 2003, 125, 13324.

18 J. An, C. M. Shade, D. A. Chengelis-Czegan, S. Petoud and N. L. Rosi, J. Am. Chem. Soc., 2011, 133, 1220.

19 A. R. Hirst, B. Escuder, J. F. Miravet and D. K. Smith, Angew. Chem., Int. Ed., 2008, 47, 8002.

20 X. Du, J. Zhou, J. Shi and B. Xu, Chem. Rev., 2015, 115, 13165.

21 K. Nishiyama, Y. Watanabe, K. Watanabe and T. Harada, Chem. Lett., 2012, 41, 1697.

22 H. Kim and J. Y. Chang, RSC Adv., 2013, 3, 1774.

23 O. Kotova, R. Daly, C. M. G. dos Santos, M. Boese, P. E. Kruger, J. J. Boland and T. Gunnlaugsson, Angew. Chem., Int. Ed., 2012, 51, 7208.

24 H. Wang, X. Li, F. Fang and Y. Yang, Dalton Trans., 2010, 39, 7294.

25 S. Banerjee, R. Kandanelli, S. Bhowmik and U. Maitra, Soft Matter, 2011, 7, 8207.

26 M. Li, Y. Wang, Y. Chen and S. A. Zhang, Photochem. Photobiol. Sci., 2014, 13, 1025.

27 T. Wang, P. Li and H. Li, ACS Appl. Mater. Interfaces, 2014, 6, 12915.

28 G. M. Peters, L. P. Skala and J. T. Davis, J. Am. Chem. Soc., 2016, 138, 134.

29 M. Yamanaka, K. Yanai, Y. Zama, J. Tsuchiyagaito, M. Yoshida, A. Ishii and M. Hasegawa, Chem.-Asian J., 2015, 10, 1299.

30 J. Takeshita, Y. Hasegawa, K. Yanai, A. Yamamoto, A. Ishii, M. Hasegawa and M. Yamanaka, Chem.-Asian J., 2017, 12, 2029.

31 Z. Li, L. E. Z. Buerkle, M. R. Orseno, K. A. Streletzky, S. Seifert, M. A. Jamieson and S. J. Rowan, Langmuir, 2009, 26, 10093. 
32 D. W. Horrocks and D. R. A. Sudnick, J. Am. Chem. Soc., 1979, 101, 334.

33 R. M. Supkowski and W. D. Horrocks, Inorg. Chim. Acta, 2002, 340, 44.

34 M. Li and P. R. Selvin, J. Am. Chem. Soc., 1995, 117, 8132.

35 M. Kawa and J. M. Fréchet, Chem. Mater., 1998, 10, 286.

36 A. Sillen and Y. Engelborghs, J. Photochem. Photobiol., A, 1998, 67, 475 .
37 M. Hasegawa, H. Ohtsu, D. Kodama, T. Kasai, S. Sakurai, A. Ishii and K. Suzuki, New J. Chem., 2014, 38, 1225.

38 K. Binnemans, Coord. Chem. Rev., 2015, 295, 1.

39 P. R. Matthes, C. J. Höller, M. Mai, J. Heck, S. J. Sedlmaier, S. Schmiechen, C. Feldmann, W. Schnick and K. MüllerBuschbaum, J. Mater. Chem., 2012, 22, 10179.

40 S. Katagiri, Y. Hasegawa, Y. Wada and S. Yanagida, Chem. Lett., 2011, 33, 1438. 